\title{
Advances in Modern Pharmacology Research of Tabanus
}

\author{
Yue Qu' ${ }^{1}$, Zhenyuan $\mathrm{Hu}^{2 *}$, Yue Zhao' \\ ${ }^{1}$ Shaanxi University of Chinese Medicine, Xianyang 712046, Shaanxi Province, China \\ ${ }^{2}$ The Affiliated Hospital of Shaanxi University of Chinese Medicine, Xianyang 712000, Shaanxi Province, China \\ *Corresponding author: Zhenyuan Hu, xyxnb3@163.com
}

\begin{abstract}
Tabanus is a traditional Chinese medicine. In recent years, various protein active components derived from the Tabanus salivary gland have been explored, and many promising components were found to have therapeutic effect. The rich active components in its salivary gland have pharmacological effects, such as anti-platelet aggregation, anti-inflammatory, anti-infection, immunosuppression, anti-angiogenic, and anti-tumor effects. This paper summarizes findings from the related articles about Tabanus in recent years with a special focus on its pharmacological effects so as to provide reference for its further development and utilization.
\end{abstract}

Keywords: Tabanus; Salivary gland; Protein active substance; Pharmacological action

Publication date: September 2021; Online publication: September 30, 2021

\section{Tabanus}

Tabanus is the whole dry female of multicarrier or other insect of the same genus of the family ophididae, diptera, Brachycera, Class Insecta. It is a traditional Chinese medicine. Tabanus, commonly called gadfly, is used to overcome blood addiction, expel blood stasis and dredge meridians. It is mainly used to cure the accumulation of blood stasis, blood storage and closure of blood stasis meridians.

\section{Various components of Tabanus}

Certain studies found that the salivary gland (SGE) of tabanus contains protein peaks with pharmacological activities ${ }^{[1.2]}$. A large number of fatty acids and other substances were identified in the components of Tabanus ${ }^{[3]}$. Jin et al. extracted mucopolysaccharide, which is the anticoagulant component, from Tabanus ${ }^{[4]}$. Jiang et al. found that gadfly is rich in copper, chromium, manganese, strontium, iron and zinc ${ }^{[5]}$.

\section{Pharmacological action of Tabanus}

It was found that cecropin-TY1, immunomodulatory protein HA and immunoregulin TPs in Tabanus SGE had strong immune and anti-inflammatory effects ${ }^{[2,6,7]}$. Immunomodulatory protein HA inhibits the maturation and phagocytosis of macrophages ${ }^{[6]}$. Tabimmunregulins increased the production of IL-10 and decreased IFN- $\gamma$ secretion ${ }^{[2]}$. Zhai found that Tabanus extract had inhibitory effect on the ear swelling in mice ${ }^{[3]}$. Li et al. found that Tabanus compound decoction can inhibit the swelling of mice, reduce the writhing times of mice, prolong the pain threshold of hot stimulation in mice, and has anti-inflammatory and analgesic effects ${ }^{[8]}$.

Tablysin ${ }^{[1]}$ and tabhibitins ${ }^{[2]}$ compete with fibrinogen to bind GPIIb/IIIa and inhibit platelet aggregation. Tabkunins can inhibit the hydrolytic activity of trypsin, thrombin, elastase and chymotrypsin on the chromogenic substrate ${ }^{[1]}$. Zhang et al. proved that vasotab TY combined with GPIIb/IIIa can easily 
pass through the lung tissue, and the liver is the main target organ ${ }^{[9]}$. The vasodilation activity of Tabanus SGEs is resistant to high temperature ${ }^{[10]}$. Another study showed that Tabanus pod may act as a blocker of integrin receptor-mediated cell adhesion ${ }^{[11]}$. Apyrase TY can inhibit adenosine diphosphate (ADP)-induced platelet aggregation ${ }^{[1,12]}$. Apyrase removes inorganic phosphorus from adenosine triphosphate (ATP) and ADP, thereby preventing platelet aggregation.

Another experiment found that antigen 5-like protein (named TabRTS) in Tabanus salivary gland can significantly inhibit angiogenesis ex vivo and in vivo ${ }^{[13]}$. TabRTS may play an anti-angiogenic role by targeting $\alpha 1 \beta 1$ integrin. Tablysin-15 can inhibit endothelial cell proliferation induced by fibroblast growth factor (FGF) and inhibit angiogenesis in vitro ${ }^{[14]}$. Deng et al. found that Tlysin-15 has anticancer effect on human melanoma cells and binds integrin $\alpha \mathrm{V} \beta 3$ to inhibit proliferation, migration and invasion of tumor cells ${ }^{[15]}$. Deng et al. found that Tlysin- 15 binds to $\alpha \mathrm{V} \beta 3$ in vitro to inhibit the proliferation, migration and invasion of breast cancer cell lines ${ }^{[16]}$. Si and Lang described the potential of MTs as biomarkers for cancer diagnosis and prognosis ${ }^{[17]}$.

Defensin-Ty1, cecropin-Ty1 and atactin-Ty3 in Tabanus SGE showed anti-bacterial activity against the tested microorganisms ${ }^{[2]}$. Cecropin-Ty1 is an adenosine monophosphate (AMP) with anti-bacterial activity ${ }^{[2]}$. Cecropin-Ty1 plays an important role in anti-inflammatory treatment of sepsis and endotoxic shock caused by Gram-negative bacterial infection ${ }^{[18]}$.

The vasoactive substance vasotab in Tabanus SGE may have a similar effect on myocardium as calcium channel antagonist ${ }^{[10]}$. Vasodilation occurs through the mechanism of strong calcium channel blocking. Takác et al. experimented with the extract of Tabanus SGE and found that SGE increased coronary blood flow and made the ventricle contract without affecting the heart rate or rhythm ${ }^{[19]}$.

Metallothionein (MTs) TY1 and TY2 of Tabanus SGE ${ }^{[2]}$ are metal binding proteins that protect cells and tissues from metal toxicity and oxidant damage ${ }^{[20]}$. Tablysin-15 significantly inhibited bone loss in mouse models and reduced the expression of osteoporosis marker genes, including MMP-9, TRAP, CTSK and $c-S r c^{[21]}$.

\section{Discussion}

In recent years, experiments on various components of Tabanus salivary gland to explore their anti-platelet aggregation, anti-inflammatory, immunosuppression, anti-angiogenic, anti-oxidation, and anti-cancer effects as well as their efficacy in the treatment of osteoporosis have laid a foundation for the further research and development of Tabanus. This also provides a new idea for drug development. The immunosuppressive active components of Tabanus salivary gland hold immense research prospect and potential, but the research on whether there are immunosuppressive components in Tabanus extract is not sufficient at present. The role of Tabanus and its salivary gland in angiogenesis is recognized. More data are still needed for their applications in treating angiogenesis diseases, including tumor, psoriasis, internal rheumatoid arthritis, and cerebral infarction. Some scholars regard its value in analgesia, but the in-depth studies on the mechanism of analgesia remain scarce. Hence, the value of Tabanus as a valuable insect traditional Chinese medicine needs to be further explored and developed.

\section{Disclosure statement}

The authors declare no conflict of interest.

\section{References}

[1] Ma D, et al., 2009, Anti-Thrombosis Repertoire of Blood-Feeding Horsefly Salivary Glands. Mol Cell Proteomics, 8(9): 2071-2079. 
[2] Xu X, et al., 2008, Toward an Understanding of the Molecular Mechanism for Successful Blood Feeding by Coupling Proteomics Analysis with Pharmacological Testing of Horsefly Salivary Glands. Mol Cell Proteomics. 7(3): 582-590.

[3] Zhai Y, 2007, Studies on the Chemical Constituents of Traditional Chinese Medicine Tabanus. Shenyang Pharmaceutical University.

[4] Jin W, et al., 2000, Extraction and Identification of Anticoagulant Substances from Tabanus. Acta Chinese Medicine and Pharmacology, 2000(03): 58-60.

[5] Jiang B, et al., 1992, Determination of Trace Elements in Five Tabanus Species. Trace Elements, 1992(01): 60-61.

[6] Chen R, et al., 2019, An Immunosuppressive Peptide from the Horsefly Inhibits Inflammation by Repressing Macrophage Maturation and Phagocytosis. J Cell Biochem. 120(8): 14116-14126.

[7] Zhao R, et al., 2009, Immunoregulatory Peptides from Salivary Glands of the Horsefly, Tabanus pleskei. Comp Biochem Physiol B Biochem Mol Biol. 154(1): 1-5.

[8] Li J, et al., 2006, Effects of Diedang Decoction on Uterine Microcirculation and its Analgesic and Anti-inflammatory Effects in Rats. Study Journal of Traditional Chinese Medicine, 2006(02): 251225.

[9] Zhang Z, et al., 2014, A Potent Anti-Thrombosis Peptide (Vasotab TY) from Horsefly Salivary Glands. Int J Biochem Cell Biol. 54: 83-88.

[10] Rajská P, et al., 2003, Vasodilatory Activity in Horsefly and Deerfly Salivary Glands. Med Vet Entomol, 17(4): 395-402.

[11] Urioste S, et al., 1994, Saliva of the Lyme Disease Vector, Ixodes dammini, Blocks Cell Activation by a Nonprostaglandin E2-Dependent Mechanism. J Exp Med. 180(3): 1077-1085.

[12] An S, et al., 2011, A Novel Allergen Tab y 1 with Inhibitory Activity of Platelet Aggregation from Salivary Glands of Horseflies. Allergy, 66(11): 1420-1427.

[13] Ma D, et al., 2010, A Horsefly Saliva Antigen 5-Like Protein Containing RTS Motif is an Angiogenesis Inhibitor. Toxicon. 55(1): 45-51.

[14] Ma D, et al., 2011, A Novel Family of RGD-Containing Disintegrins (Tablysin-15) from the Salivary Gland of the Horsefly Tabanus Yao Targets $\alpha \mathrm{IIb} \beta 3$ or $\alpha \mathrm{V} \beta 3$ and Inhibits Platelet Aggregation and Angiogenesis. Thromb Haemost. 105(6): 1032-1045.

[15] Deng Z, et al., 2020, Disintegrin Tablysin-15 Suppresses Cancer Hallmarks in Melanoma Cells by Blocking FAK/Akt/ERK and NF-кB Signaling. Curr Cancer Drug Targets, 20(4): 306-315.

[16] Deng Z, et al., 2019, The Anticancer Properties and Mechanism of Action of Tablysin-15, the RGDContaining Disintegrin, in Breast Cancer Cells. Int J Biol Macromol, 129: 1155-1167.

[17] Si M, Lang J, 2018, The Roles of Metallothioneins in Carcinogenesis. J Hematol Oncol, 11(1): 107.

[18] Wei L, et al., 2015, A Potent Anti-Inflammatory Peptide from the Salivary Glands of Horsefly. Parasit Vectors, 8: 556.

[19] Takác P, et al., 2006, Vasotab, a Vasoactive Peptide from Horse Fly Hybomitra bimaculata (Diptera, Tabanidae) Salivary Glands. J Exp Biol, 209(Pt 2): 343-352.

[20] Irie Y, et al., 2004, Expression of Neuronal Growth Inhibitory Factor (Metallothionein-III) in the Salivary Gland. Physiol Res, 53(6): 719-723.

[21] Zeng Q, et al., 2020, Tablysin-15 Inhibits Osteoclastogenesis and LPS-Induced Bone Loss via Attenuating the Integrin $\alpha v \beta 3$ Pathway. Chem Biol Interact, 327: 109179. 